\title{
Innovating to achieve service excellence in Alberta Health Services
}

\author{
Kathryn A. Ambler MSc, Marc A. Leduc MA, Patty Wickson MBA
}

— Cite as: CMAJ 2019 December 4;191(Suppl 1):S52-3. doi: 10.1503/cmaj.190598

I

nnovation is essential for any learning, high-performing health system. It can enhance patient outcomes, improve patient and provider experiences, fulfil unmet care and service needs, eliminate redundant processes and technologies, and promote financial sustainability. Innovation can also be difficult, ${ }^{2}$ particularly when adopting initiatives at scale, which is necessary to realize the value of specific innovations. Rigorously evaluating, implementing and sustaining innovation necessitates fast-paced, failure-prone iteration while coordinating across organizational silos and overcoming change fatigue. In health care, challenges can be seen as innovation-related opportunity costs and investment needs compete for scarce resources. In response, Alberta Health Services (AHS) has established teams, resources and a clear process to ensure that Albertans benefit from health innovation. This strategy aims to leverage innovation to achieve service excellence and ensure a patient-focused, quality health system that is accessible and sustainable for all.

In 2012, the launch of the Strategic Clinical Networks (SCNs) ${ }^{3}$ created a new platform for innovation in Alberta. The networks bring together diverse stakeholders to identify pressing problems, design and test solutions within clinical environments, and spread proven innovations across the province. In 2017, the Innovation, Evidence and Impact Team was retooled to be a coordinating hub that facilitates health innovation (new and improved way of doing valued things ${ }^{4}$ in the fields of medical devices, diagnostics, information technology, methods of treatment and models of care). The team provides education and system navigation for both internal and external clients. Expertise is also offered to AHS staff and clinicians to support definition of value propositions; generation, synthesis and assessment of evidence; health economic and program evaluation; benefit and cash flow forecasting and measurement; and feasibility analyses. The team also monitors and responds to emerging innovations in areas such as artificial intelligence, virtual care and personalized health, and works with the SCNs to leverage organizational assets (e.g., a provincial Contracting Procurement and Supply Management Department and a single clinical information system). The collaborative efforts of the SCNs and the team create a synergy that capitalizes on their unique structures and talent and catalyzes innovation across the health system.

\section{KEY POINTS}

- Innovation, when aligned with health system priorities and implemented at scale, can improve outcomes, patient and provider experience, and financial sustainability of the health system.

- The Strategic Clinical Networks and Alberta Health Services' Innovation, Evidence and Impact Team prioritize, evaluate and advance health innovations that respond to the needs of Albertans. Together, they build capacity and competency within the organization to realize the value of innovation through adoption at scale.

- The Innovation-to-Action Lifecycle is an evidence-informed process that helps staff, clinicians and innovators move innovations from an idea, through testing, to adoption at scale (if appropriate) and evaluation to ensure adequate return on investment.

- System-level supports are necessary to innovate successfully. These include committed executive sponsor(s), engaged stakeholders representing all relevant perspectives, dedicated resources and a culture supportive of innovation.

Once established, the SCNs and the team worked to bolster capacity and capability for innovation across the system. Recognizing that the skills of members of the SCNs and mindsets around innovation are different, the Innovation, Evidence and Impact Team hosted workshops to clarify roles and expectations, build knowledge and expertise, and foster a culture of innovation. Education sessions were provided on topics such as economic analysis and criteria for effective decision-making. Linkages between the SCNs, the team and partners outside of AHS were strengthened.

At an organizational level, key issues, such as better hand-offs across the system, senior leader champions and dedicated resources, needed strengthening. A communication strategy was developed, and the team engaged with operational, clinical support and corporate divisions of AHS to address these issues. Furthermore, AHS and its partners created competitive funding programs to enable the SCNs to develop, evaluate and implement innovations of value. In 2020, experts from the Northern and Southern Alberta Institutes of Technology will assess the effect these efforts have had on innovation capacity, competencies and culture within SCNs, the organization and the broader provincial health innovation ecosystem. 
A standard process was also required to give innovators and their sponsors more clarity on how to produce evidence of value, anticipate the needs of future decision-makers when seeking to scale ideas, and to mitigate common business and clinical risks that innovators face in health care. ${ }^{5}$ Input from the SCNs helped the team to design and deploy the Innovation-to-Action Lifecycle (Appendix 1, available at www.cmaj.ca/lookup/suppl/doi:10.1503/ cmaj.190598/-/DC1). Incorporating concepts and suggestions ${ }^{6}$ from Canadian leaders, such as MaRS EXCITE, Ontario's Office of the Chief Health Innovation Strategist and Innovation Boulevard, the lifecycle provides a consistent and transparent means to assess innovations that are proposed to AHS ("pushed innovation") and to enable AHS to seek innovative solutions ("innovation pull"). This ensures that factors critical for success ${ }^{7,8}$ are addressed in a stepwise fashion. A match is made between priority problems in the health system and innovative solutions, committed sponsors who accept accountability are engaged, and then evidence generation and assessment proceeds to inform decisions and set the stage to facilitate adoption as appropriate.

The Innovation-to-Action Lifecycle enables both reactive and proactive tactics to innovation procurement. ${ }^{9}$ In the most passive approach, pushed innovations submitted to the Innovation, Evidence and Impact Team are forwarded to internal stakeholders for vetting. Since its launch in early 2018,81 innovations have been processed through the lifecycle. Interest was expressed in about $4 \%$ of submissions. In most cases, stakeholders found that proposed innovations did not address a problem in the health system, or the problem did not have sufficient priority to merit the work required to explore the possibility of uptake. Overall, however, the lifecycle has provided a standardized, high-capacity means for AHS to consider pushed innovation, while increasing the speed and consistency of decision-making and providing important feedback to innovators about the rationale behind decisions.

To pull innovation into the health system, the Innovation, Evidence and Impact Team has developed targeted methods to apply the lifecycle. Events such as reverse trade shows, ${ }^{10}$ discovery days and early market-engagement sessions provide an opportunity to convene industry and health system stakeholders, communicate narrowly scoped priorities and showcase related innovations. These catalytic activities have proven fruitful, leading to follow-on work such as real-world evidence trials, data-access agreements and, in 1 instance, adoption of an innovation by the Alberta Aids to Daily Living program. Although resource intensive, these directed approaches offer the most promise for finding or co-designing innovative solutions, because they are characterized by a high degree of commitment from all vested executive sponsors and stakeholders, alignment with the SCNs' Transformational Roadmaps and current activities, engagement from innovators and industry partners, and eligibility for innovation-specific funding opportunities.

Realizing the value of innovation requires a way to find and test emerging solutions efficiently and effectively and add to or replace current practices with the best of these solutions. Although the strategic investments, changes and infrastructure put in place through the SCNs and the Innovation, Evidence and Impact Team have increased organizational competency and capacity, there is still work to do to. Future efforts will focus on increasing throughput of the Innovation-to-Action Lifecycle, enhancing the nimbleness and agility with which innovations are processed, and advancing the adoption rate of pushed and pulled innovation. As a learning health care organization, AHS is committed to building its capabilities to discover, adopt and scale innovative solutions to achieve its vision of "Healthy Albertans. Healthy Communities. Together."

\section{References}

1. Haughom J. Innovation in healthcare: why it's needed and where it's going. Salt Lake City (UT): Health Catalyst; 2014. Available: www.healthcatalyst.com/ innovation-in-healthcare-why-needed-where-going (accessed 2019 Sept. 23).

2. Côté-Boileau É, Denis JL, Callery B, et al. The unpredictable journeys of spreading, sustaining and scaling healthcare innovations: a scoping review. Health Res Policy Syst 2019;17:84.

3. Yiu V, Belanger F, Todd K. Alberta's Strategic Clinical Networks: Enabling health system innovation and improvement. CMAJ 2019;191(Suppl 1):S1-3.

4. AHS Strategy for clinical health research, innovation and analytics, 2015-2020. Edmonton: Alberta Health Services. Available: www.albertahealthservices.ca/ assets/info/res/if-res-strat-doc.pdf (accessed 2019 July 9).

5. Lennon MR, Bouamrane MM, Devlin AM, et al. Readiness for delivering digital health at scale: lessons from a longitudinal qualitative evaluation of a national digital health innovation program in the United Kingdom. J Med Internet Res 2017;19:e42.

6. CMA Health Summit 2018: summary report. Ottawa: Canadian Medical Association; 2019. Available: https://cmahealthsummit.ca/app/uploads/2019/02/HS-Board -Report_Final.pdf (accessed 2019 Sept. 23).

7. Greenhalgh T, Wherton J, Papoutsi C, et al. Beyond adoption: a new framework for theorizing and evaluating nonadoption, abandonment, and challenges to the scale-up, spread, and sustainability of health and care technologies. J Med Internet Res 2017;19:e367.

8. Nolte E. How do we ensure that innovation in health service delivery and organization is implemented, sustained and spread? Geneva: World Health Organization; 2018. Available: www.euro.who.int/_data/assets/pdf_file/0004/380731/pb-tallinn -03-eng.pdf?ua=1 (accessed 2019 Sept. 23).

9. BPS primer on innovation procurement interim. Toronto: Supply Chain Ontario; 2014. Available: www.doingbusiness.mgs.gov.on.ca/mbs/psb/psb .nsf/0/df7388300f40aec68525814d004a00bf/\$FILE/BPS_Primer_on_Innovation _Procurement_Interim.pdf (accessed 2019 Sept. 23).

10. Partnering for health system innovation in Alberta: summary report from SCN/ MEDEC Engagement - final report. Edmonton: Institute of Health Economics; 2015. Available: www.ihe.ca/publications/partnering-for-health-system-innovation -in-alberta-summary-report-from-scn-medec-engagement-ndash-final-report (accessed 2019 Sept. 23).

Competing interests: Kathryn Ambler, Marc Leduc and Patty Wickson are employees of Alberta Health Services. No other competing interests were declared.

This article has been peer reviewed.

Affiliations: Innovation, Evidence \& Impact Team (Ambler, Wickson), and Health Evidence and Innovation (Leduc), Alberta Health Services, Edmonton, Alta.

Contributors: All of the authors made substantial contributions to the conception, design and implementation of the work. Kathryn Ambler wrote the first draft of the manuscript, and Marc Leduc and Patty Wickson reviewed and revised it in collaboration with Kathryn Ambler. All of the authors gave final approval of the version to be published and agreed to be accountable for the work described.

Acknowledgements: The authors thank Allison Strilchuk (scientific writer with the Pan-SCN team) for providing thoughtful feedback and revisions on this manuscript.

Correspondence to: Marc Leduc, marc.leduc@ahs.ca 